\title{
Cervical cytology and histology in the context of a screening programme
}

\author{
Sanjiv Manek \\ Gynaecological Pathologist \\ Oxford \\ UK
}

In the UK, the national cervical cancer screening programme is probably the most established, robust and rigorous of all screening programmes that has evolved over 25 years. It has seen a multitude of changes based on evidence obtained from a vast amount of research and observations, as well as from advances in technology. This success has led to a definite impact on cervical cancer incidence with reduced mortality and morbidity related to cervical cancer. More recently, the changes introduced to the programme have been exponential and in the UK we are at the brink of a new direction and a new style of provision of the screening programme. Throughout the years, there has been a continued effort to understand morphological aspects of the cytology and histology in cervical neoplasia and many new entities have been recognised or variations of known entities realised. The intricate measures to ensure quality in the screening programme have led to close working relationships between cytologists, histopathologists, colposcopists, and now, molecular biologists. The UK cervical cancer screening programme (CCSP) is likely to change significantly in the next few years, and although reduced in quantity, cytology will still remain an important aspect of it. The importance of histopathology is unlikely to change, but more onus will be placed on the molecular biologists and colposcopists.

In order to discuss and understand the importance of cytology and histology in the cervical screening programme and the absolute requirement of correlation between the two, it is important first to appreciate how the UK CCSP has evolved and how it has taken with it the evolution of new technologies in cytology leading to improved correlation between histology and cytology and quality assurance with obvious benefits to the patient.

\section{Milestones in the UK CCSP}

1987/8 - $\quad$ Launch of formal programme.

$1989 \quad$ - Introduction of 3-tier system of reporting

(Code 7 - moderate dyskaryosis).

$1994 \quad-\quad$ Formal introduction and definition of the borderline category

(Code 8).

$1996 \quad$ - $\quad$ Formal introduction of review of screened slides.

$1998 \quad$ - Introduction of quality assurance measures including external quality assurance tests.

$2000 \quad-\quad$ Achievable Benchmark Criteria (ABC) I.

$2002 \quad-\quad$ Terminology refinement.

$2003 \quad-\quad$ Achievable Benchmark Criteria (ABC) II

$2005 \quad$ - Introduction of the invasive cancer audit

$2006 \quad$ - $\quad$ Changes in age ranges for screening and follow-up protocols. 
$\begin{array}{lll}2007 & - & \text { Liquid-based cytology }( \pm \text { HPV vaccine }) . \\ 2011 & - & \text { Targets for quick turnaround. } \\ 2012 & - & \text { Introduction of HPV testing as triage and test of cure } \\ \text { Future } & - & \text { Networks, automation in screening and HPV testing as a primary screening tool. }\end{array}$

Of these milestones, the major ones have been: 1) the ABC I and II documents which have introduced not only the finer details of morphology in cervical cytology and histology, but also guidelines for reporting within certain parameters; 2) the terminology refinement; 3) the change in screening age ranges; 4) LBC; and 5) HPV testing. Each time such changes were introduced, there were changes in reporting practice also seen and considered necessary and the management of patients better defined and tailored. As a result, there has been a vast amount of literature on morphology in conventional cytology and LBC, as well as literature on outcomes.

\section{$\underline{\text { Cytology }}$}

The main categories of reporting currently are:

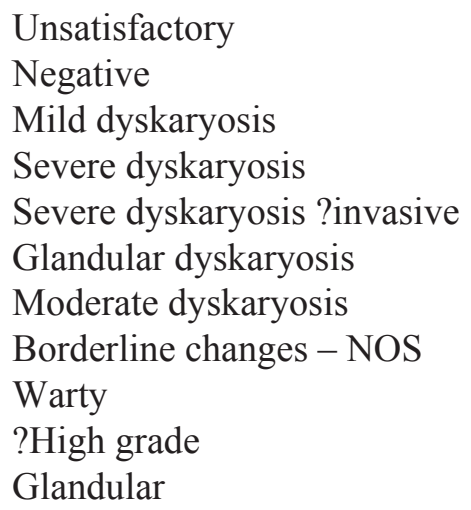

These categories were very well defined in conventional cytology and for many, particularly for severe dyskaryosis, many patterns have been recognised through careful observation and regular audits. These categories are equally reproducible in LBC and, in fact, are somewhat easier to diagnose. With the advent of HPV testing, some of these categories will be refined in due course.

\section{Histology}

The UK NHS CSP national office has published multiple documents on guidelines and amongst these, there are two on the histopathology of cervical lesions. The main categories to recognise are:

HPV changes

Cervical Intraepithelial Neoplasia (CIN) 1, 2 and 3

CIN 3 with crypt involvement

Low and high grade cervical glandular intraepithelial neoplasia (CGIN)

Early stromal invasion

$>$ Stage 1A2 carcinomas and all the subtypes

Stratified mucinous intraepithelial lesion (SMILE)

Tubo-endometrioid metaplasia 
In the UK, the retention of the 3-tier system of reporting in cytology and histology has allowed detailed correlation between the two. In everyday practice, correlation plays a very important role in providing the best management to a patient and it helps to enhance failsafe procedures and to improve quality assurance measures.

\section{Correlation in cervical pathology}

Much of cervical biopsy reporting is dependent on correlation with referral or recent cytology. An adequate request form should state the expected degree of abnormality by mentioning the referral cytology findings. This can be accompanied by the colposcopic impression which is useful but less important where correlation is concerned. If clinical details are inadequate or variable, it is good practice to have the past history readily available at the time of reporting both histology and cytology. This allows appropriate and safe prospective reporting. The typical cervical histology report should have a comment on correlation, and where possible, all non-correlation cases should be worked up prospectively prior to issuing the final report. This would involve examining multiple levels of the biopsy, immunohistochemistry (p16, bcl2, etc) and reviewing the cytology. If the cytology is confirmed, then the appropriate management could be advised in the histology report and this includes repeat colposcopy and biopsy. Where relevant, cases should be discussed at dedicated colposcopy MDT meetings. The review of the cytology could be used as a learning exercise when there is overcall and undercall of abnormalities. Examining levels could mean cutting through the block if necessary, particularly for high grade lesions.

If the cytology is high grade and confirmed by more than one person, and the subsequent histology is repeatedly lower grade or negative, follow up should be according to cytology in case of vaginal or high grade canal disease.

Problems arise when cytology and histology are divorced and correlation exercises are carried out retrospectively, often with significant delays. Patients may slip through failsafe, which can lead to delayed diagnosis.

The commonest situation of non-correlation is where the cytology has been reported as high grade dyskaryosis and the subsequent biopsy is negative or only low grade. If levels do not reveal any correlating lesions, the possible reasons to consider are high canal disease, vault disease, tiny lesions and inadequate colposcopy. In a significant proportion of cases, the expected lesion is discovered in subsequent biopsies. Sometimes a loop excision is performed as treatment after a biopsy diagnosis of a high grade lesion and there is no correlating lesion. Once again, the possibility of a tiny lesion (treated by the biopsy) needs to be considered if multiple levels still do not reveal the expected histology.

Undercall in cytology is usually due to misinterpretation of crowded groups or missing single dyskaryotic cells that may be small or keratinised, dark or pale. Occasionally immature metaplastic cells can be overlooked.

Overcall in cytology is usually due to mistaking immature or reactive metaplastic cells as high grade dyskaryosis and crowded groups as microbiopsies of CIN 3.

Correlation with cytology reported as borderline is interesting. Borderline NOS or borderline reactive usually correlates with inflammatory changes or low grade CIN. Occasionally, a high grade lesion is seen and review of the cytology may show high grade dyskaryotic features. Borderline glandular changes may yield reactive features or CGIN on biopsy. It is a matter of experience and constant review of such cases to gain the confidence to report the cytology as either negative or glandular dyskaryosis.

When LBC was introduced, there was a high incidence of reporting borderline ?high grade dyskaryosis. Most of these turn out to be CIN 2/3 on histology. With time and with learning from consistent reviews, most of these can be reclassified as high grade dyskaryosis.

With HPV testing, a new type of correlation challenge is being encountered. What is to be expected when the cytology is negative and high risk HPV testing is positive? Many biopsies are negative (correlate with cytology) but some are high grade. Review of cytology in such high grade cases tends to show negative features in most and abnormalities in only a few. In negative biopsy cases, follow up would be return to normal recall unless the colposcopist decides otherwise. The other conundrum that has emerged is where the first cytology

14. Kongres udruženja patologa i citologa Srbije sa međunarodnim učešćem, Beograd 14-16 juna, 2012

14 th Congress of Serbian Association of Pathologists and Cytologists with international participation, Belgrade 14-16 June, 2012 
after a treatment is negative but HPV testing is positive rendering it necessary to colposcope. In many such cases, the colposcopy is also negative. The type of further management is then decided by the colposcopist.

One more aspect of correlation in cervical pathology is in cervical cancer staging. Beyond stage 1A2 tumours, which depend on measurements, the more reliable modality for staging is imaging (MRI) and clinical, partly because of the three dimensional nature of lesions. There are a significant number of cases where staging by pathology is much lower than that shown by imaging; hence, final staging has to be left to multidisciplinary team meetings.

\section{$\underline{\text { Litigations }}$}

Over the years, there have been numerous litigation cases in cervical cancer development. Some are related to surgical issues but most are for possible errors in cytology reporting and occasionally for histology reporting. In the UK there have been famous instances of litigation and these have led to some of the changes in the CCSP mentioned above. In particular, the invasive cancer audit was introduced as an educational tool to understand why cancers develop despite women adhering to the screening programme. One has to appreciate that with human interpretation there will be a small percentage of false negative cytology but what is really important in this situation is to know what leads to false negative cytology. The audit is a very useful tool for this and with its use, the UK cytopathologists have seen some common patterns and variations in morphology accountable for false negative reporting. These include the categories of scanty dyskaryosis, small keratinised cells, small cell (dark) dyskaryosis, pale dyskaryosis and microbiopsies. The 'litigation' cell is often the small dyskaryotic cell!

\section{The future}

There will be a reduction in the amount of cytology required as a result of HPV testing, to the point where HPV testing may become the primary screening tool and cytology, the triage test. Much of the cytology screening will be automated, leaving only a small amount for human interpretation. All the processing and screening is likely to be carried out in hyper-laboratories. There will be changes in correlation processes because histology will need to be matched with the HPV test result rather than with the cytology in low grade lesions. Despite all the major overhauls, the UK CCSP is likely to remain cost-effective with major benefits which, when the effects of the HPV vaccine are felt, should see a dramatic reduction in the cervical cancer incidence in the UK whilst avoiding major surgical interventions and hence other complications.

\section{$\underline{\text { References }}$}

1. www.cancerscreening.nhs.uk/cervical

2. www.britishcytology.org.uk 